PROCEEDINGS OF THE

AMERICAN MATHEMATICAL SOCIETY

Volume 135, Number 8, August 2007, Pages 2461-2470

S 0002-9939(07)08940-X

Article electronically published on April 5, 2007

\title{
HARDY SPACES AND PARTIAL DERIVATIVES OF CONJUGATE HARMONIC FUNCTIONS
}

\author{
ANATOLY RYABOGIN AND DMITRY RYABOGIN
}

(Communicated by Mei-Chi Shaw)

\begin{abstract}
In this paper we give necessary and sufficient conditions for a harmonic vector and all its partial derivatives to belong to $H^{p}\left(\mathbf{R}_{+}^{n+1}\right)$ for all $p>0$.
\end{abstract}

\section{IntRodUCTION AND STATEMENTS OF MAIN RESULtS}

In this article we study the following problem: what can we say about conjugate harmonic functions in $\mathbf{R}_{+}^{n+1}=\mathbf{R}^{n} \times(0, \infty)$, provided we are given certain restrictions, imposed on partial derivatives of a harmonic vector $F$,

$$
F=\left(U(x, y), V_{1}(x, y), V_{2}(x, y), \ldots, V_{n}(x, y)\right), \quad(x, y) \in \mathbf{R}_{+}^{n+1} .
$$

We refer the reader to the classical works [11, [3], 13], [15], [5], [2], 4], [16] for the history and different results related to this problem and the classes $S^{p}\left(\mathbf{R}_{+}^{n+1}\right)$, $h^{p}\left(\mathbf{R}_{+}^{n+1}\right), H^{p}\left(\mathbf{R}_{+}^{n+1}\right.$ ) (all definitions are given in Section 2 ).

We give necessary and sufficient conditions for a harmonic vector and all its partial derivatives up to the order $k$ to belong to $H^{p}\left(\mathbf{R}_{+}^{n+1}\right), p>0$. Our main result is

Theorem 1. Let $0<p<q$. The harmonic vector $F=\left(U, V_{1}, \ldots, V_{n}\right)$ and all its partial derivatives of order $\leq k$ belong to $H^{r}, p \leq r \leq q$, if and only if

$$
\text { 1) } \left.M_{p}(y+1, F) \leq C, \quad 2\right) \int_{\mathbf{R}^{n}}\left(\sup _{\eta \geq y}\left|D_{n+1}^{k} U(x, \eta)\right|\right)^{q} d x \leq C .
$$

The case $p<(n-1) / n$ leads to additional technical difficulties, since $|F|^{p}$ is subharmonic, provided $p \geq(n-1) / n$, 13. One of the methods of the proof is the application of classes $S^{p}\left(\mathbf{R}_{+}^{n+1}\right)$ together with the Lagrange mean-value theorem. We also use the boundary behaviour of the conjugate harmonic functions. We note that the first condition in (1) is natural, not only due to the decomposition of the function into two parts, " $S^{p}\left(\mathbf{R}_{+}^{n+1}\right)$ " and " $H^{p}\left(\mathbf{R}_{+}^{n+1}\right)$ " (see Section 3, proof of Theorem [5), but in fact, it, together with the second condition, implies $M_{p}(y+$ $\left.y_{0}, F\right) \leq C\left(y_{0}\right) \forall y_{0}>0$ (see Section 4, Lemma 7).

The paper is organized as follows. In Section 2 we give all necessary definitions and auxiliary results used in the sequel. Section 3 is devoted to the results needed

Received by the editors January 31, 2006.

2000 Mathematics Subject Classification. Primary 30E25; Secondary $42 B 25$.

Key words and phrases. Hardy spaces, subharmonic functions.

(C)2007 American Mathematical Society 
for the proof of Theorem 1 and in Section 4 we prove Theorem 1 For convenience of the reader we split our proofs into elementary lemmata.

\section{Auxiliary Results}

We begin with the definition of the classes $S^{p}\left(\mathbf{R}_{+}^{n+1}\right)$ and $h^{p}\left(\mathbf{R}_{+}^{n+1}\right)$.

Let $U(x, y)$ be a harmonic function in $\mathbf{R}_{+}^{n+1} \equiv \mathbf{R}^{n} \times(0, \infty)$. We say that the vector function $V(x, y)=\left(V_{1}(x, y), \ldots, V_{n}(x, y)\right)$ is the conjugate of $U(x, y)$ in the sense of M. Riesz [12, 14], if $V_{k}(x, y), k=1, \ldots, n$ are harmonic functions, satisfying the generalized Cauchy-Riemann conditions:

$$
\frac{\partial U}{\partial y}+\sum_{k=1}^{n} \frac{\partial V_{k}}{\partial x_{k}}=0, \quad \frac{\partial V_{i}}{\partial x_{k}}=\frac{\partial V_{k}}{\partial x_{i}}, \quad \frac{\partial U}{\partial x_{i}}=\frac{\partial V_{i}}{\partial y}, \quad i \neq k, k=1, \ldots, n .
$$

If $U(x, y)$ and $V(x, y)$ are conjugate in $\mathbf{R}_{+}^{n+1}$ in the above sense, then the vector function

$$
F(x, y)=(U(x, y), V(x, y))=\left(U(x, y), V_{1}(x, y), \ldots, V_{n}(x, y)\right)
$$

is called a harmonic vector.

Define

$$
M_{p}(y)=M_{p}(y, F)=\left\{\int_{\mathbf{R}^{n}}|F(x, y)|^{p} d x\right\}^{1 / p}, \quad p>0 .
$$

Definition ([1, 7]). We say that $F(x, y) \in S^{p}\left(\mathbf{R}_{+}^{n+1}\right), p>0$ if for any $y_{0}>0$ there exists a constant $C\left(y_{0}, F\right)$ such that $\forall y \geq y_{0}, M_{p}(y, F) \leq C\left(y_{0}\right)$. In particular, if $C$ is independent of $y_{0}$, then $F(x, y) \in h^{p}\left(\mathbf{R}_{+}^{n+1}\right)$.

Now we define the space $H^{p}\left(\mathbf{R}_{+}^{n+1}\right)$. We follow the work of Fefferman and Stein 44. Let $U(x, y)$ be a harmonic function in $\mathbf{R}_{+}^{n+1}$, and let $U_{j_{1} j_{2} j_{3} \ldots j_{k}}$ denote a component of a symmetric tensor of rank $k, 0 \leq j_{i} \leq n, i=1, \ldots, n$. Suppose also that the trace of our tensor is zero, meaning

$$
\sum_{j=0}^{n} U_{j j j_{3} \ldots j_{k}}(x, y)=0, \quad \forall j_{3}, \ldots, j_{k} .
$$

The tensor of rank $k+1$ can be obtained from the above tensor of rank $k$ by passing to its gradient:

$$
U_{j_{1} j_{2} \ldots j_{k} j_{k+1}}(x, y)=\frac{\partial}{\partial x_{j_{k+1}}}\left(U_{j_{1} j_{2} j_{3} \ldots j_{k}}(x, y)\right), \quad x_{0}=y, 0 \leq j_{k+1} \leq n .
$$

Definition (4]). We say that $U(x, y) \in H^{p}\left(\mathbf{R}_{+}^{n+1}\right), p>0$, if there exists a tensor of rank $k$ of the above type with the properties:

$$
U_{0 \ldots 0}(x, y)=U(x, y), \quad \sup _{y>0} \int_{\mathbf{R}^{n}}\left(\sum_{(j)} U_{(j)}^{2}(x, y)\right)^{p / 2} d x<\infty, \quad(j)=\left(j_{1}, \ldots j_{k}\right) .
$$

It is well known that the function $\left(\sum_{(j)} U_{(j)}^{2}(x, y)\right)^{p / 2}$ is subharmonic for $p \geq p_{k}=$ $(n-k) /(n+k-1) ;$ see [3, 4], 14]. 
We will use the "radial" and nontangential maximal functions:

$$
F^{+}(x)=\sup _{y>0}|F(x, y)|, \quad N_{\alpha}(F)\left(x^{0}\right)=\sup _{(x, y) \in \Gamma_{\alpha}\left(x^{0}\right)}|F(x, y)| .
$$

Here

$$
\Gamma_{\alpha}\left(x^{0}\right)=\left\{(x, y) \in \mathbf{R}_{+}^{n+1}:\left|x-x^{0}\right|<\alpha y\right\}, \quad \alpha>0,
$$

is an infinite cone with vertex at $x^{0}$. It is well known 4 that

$$
F(x, y) \in H^{p}\left(\mathbf{R}_{+}^{n+1}\right) \Longleftrightarrow N_{\alpha}(F)(x) \in L^{p} \Longleftrightarrow F^{+}(x) \in L^{p}, p>0 .
$$

We also define the weak maximal function

$$
W F(x, y)=\sup _{\zeta \geq y}|F(x, \zeta)|, \quad y>0 .
$$

The above expression is understood as follows: we fix $x$, and for fixed $y$ we find the supremum over all $\zeta \geq y$.

We will repeatedly use the following results.

Lemma 1 (4], p. 173). Suppose $u(x, y)$ is harmonic in $\mathbf{R}_{+}^{n+1}$, and for some $p, 0<$ $p<\infty$,

Then

$$
\sup _{y>0} \int_{\mathbf{R}^{n}}|u(x, y)|^{p} d x<\infty .
$$

$$
\sup _{x \in \mathbf{R}^{n}}|u(x, y)| \leq A y^{-n / p}, \quad 0<y<\infty .
$$

Theorem 2 ([], p. 268). Let $0<p \leq 1, k \in \mathbf{N}$, and let $u: \mathbf{R}_{+}^{n+1} \rightarrow \mathbf{R}$ be $a$ harmonic function such that

$$
u(x, t) \Rightarrow_{t \rightarrow \infty}^{x} 0, \quad K_{k, p} \equiv \int_{\mathbf{R}_{+}^{n+1}} t^{k p-1}\left|D_{n+1}^{k} u(x, t)\right|^{p} d x d t<C .
$$

Then $u(x, 0)=\lim _{t \rightarrow 0+} u(x, t)$ exists for almost all $x \in \mathbf{R}^{n}$, and for all $t \geq 0$,

$$
\int_{\mathbf{R}^{n}}|u(x, t)|^{p} d x \leq A C(k, n, p) K_{k, p} .
$$

Theorem 3 ([5], p. 269). Let $m \in \mathbf{N}, p \geq(n-1) /(m+n-1)$ (if $n=1$ we suppose $p>0)$, and let $u: \mathbf{R}_{+}^{n+1} \rightarrow \mathbf{R}$ be harmonic. Then, for all $t>0$,

$$
\int_{\mathbf{R}^{n}}\left|\nabla^{m} u(x, t)\right|^{p} d x \leq A(m, n, p) t^{-m p-1} \int_{t / 2}^{3 t / 2} d s \int_{\mathbf{R}^{n}}|u(x, t)|^{p} d x .
$$

Corollary 1 ([5, p. 270). Let $p, m$ be as in Theorem [3, let $b>0$, and let $u$ : $\mathbf{R}_{+}^{n+1} \rightarrow \mathbf{R}$ be a harmonic function such that for all $t>0$,

$$
\int_{\mathbf{R}^{n}}|u(x, t)|^{p} d x \leq C t^{-b} .
$$

Then

$$
\int_{\mathbf{R}^{n}}\left|\nabla^{m} u(x, t)\right|^{p} d x \leq A(b, m, n, p) C t^{-b-m p} \quad(t>0) .
$$


In fact, the choice of $p$ in Theorem 2 and Corollary 1 may be independent of $m$ (see Lemma 2).

Theorem 4 ([10]). Let $p>0$ and let $F(x, y)=\left(U, V_{1}, \ldots, V_{n}\right)$ be a harmonic vector satisfying
1) $V_{i}(x, y) \Rightarrow_{y \rightarrow \infty}^{x} 0, i=1, \ldots, n$,
2) $M_{p}(y, U) \leq C$,
3) $|U| \leq C$

Then $F \in H^{r}, r>p$.

Notation. We denote by $D_{i}^{k} f(x, y)$ the partial derivative of the function $f$ of order $k$ with respect to $x_{i}, i=1,2, \ldots, n+1$. $M(f)(x)$ denotes the usual HardyLittlewood maximal function of $f(x)$. The notation $f(x, y) \Rightarrow_{y \rightarrow \infty}^{x} 0$ means that $f(x, y)$ converges to 0 uniformly with respect to $x$, provided $y \rightarrow \infty, \nabla^{k} f(x)=$ $\left(\frac{\partial^{k} f(x)}{\partial x_{1}^{k}}, \ldots, \frac{\partial^{k} f(x)}{\partial x_{n}^{k}}\right)$. Everywhere below the constants $A(k, n), C, K$ depend only on the parameters pointed out in parentheses and may be different from time to time.

\section{Auxiliary lemmata for the proof of Theorem 1}

The main results of this section are Theorem 5 and Theorem 7 , Our first auxiliary result shows that in Theorem 3 and Corollary 1 the choice of $p>0$ may be independent of $m \in \mathbf{N}$. We include it here for the convenience of the reader.

Lemma 2. Let $p>0$ and let $F=\left(U, V_{1}, \ldots, V_{n}\right)$ be such that $V_{i} \Rightarrow_{y \rightarrow \infty}^{x} 0, i=$ $1, \ldots, n, M_{p}(y, U) \leq C$. Then

$$
M_{p}\left(y, \nabla^{k} F\right) \leq A C y^{-k}, \quad k \in \mathbf{N} .
$$

Proof. By induction on $k$. Let $k=1$. Fix $p>0$ and let $l=\inf \{j \in \mathbf{N}: p \geq$ $(n-1) /(j+n-1)\}$. Let $\phi_{i j}(x, y)$ be a coordinate of $\nabla V_{i}(x, y), j=1, \ldots, n+1$, $x_{n+1}=y, i=0, \ldots, n, V_{0}=U$. Since $\nabla V_{i}(x, y) \Rightarrow_{y \rightarrow \infty}^{x} 0$, we may use the following relation (see [5] or [4):

$$
\begin{aligned}
\phi_{i j}(x, y) & =\frac{1}{(2 l-2) !} \int_{y}^{\infty}(s-y)^{2 l-2} D_{n+1}^{2 l-1} \phi_{i j}(x, s) d s \\
& =\frac{1}{(2 l-2) !} \int_{0}^{\infty} s^{2 l-2} D_{n+1}^{2 l-1} \phi_{i j}(x, s+y) d s .
\end{aligned}
$$

We have $\left|\phi_{i j}(x, y)\right| \leq h_{i j}(x, y)$, where

$$
h_{i j}(x, y) \equiv \frac{1}{(2 l-2) !} \int_{0}^{\infty} s^{2 l-2}\left|\nabla^{l} D_{n+1}^{l-1} \phi_{i j}(x, s+y)\right|^{p} d s .
$$

Theorem 3 of [5] implies (take $w=\nabla^{l} D_{n+1}^{l-1} \phi_{i j}, a=2 l-1, A=A(l, n, p)$ ),

$$
\int_{\mathbf{R}^{n}}\left|\phi_{i j}(x, y)\right|^{p} d x \leq \int_{\mathbf{R}^{n}}\left|h_{i j}(x, y)\right|^{p} d x \leq A \int_{0}^{\infty} s^{(2 l-1) p-1} d s \int_{\mathbf{R}^{n}}\left|\nabla^{l} D_{n+1}^{l-1} \phi_{i j}(x, s+y)\right|^{p} d x .
$$

Since $D_{n+1}^{l-1} \phi_{i j}(x, y)$ is the $l$-th derivative of $V_{i}$, we use Theorem 3 to get

$$
\int_{\mathbf{R}^{n}}\left|\nabla^{l} D_{n+1}^{l-1} \phi_{i j}(x, y)\right|^{p} d x \leq \int_{\mathbf{R}^{n}}\left|\nabla^{2 l} F(x, y)\right|^{p} d x \leq C y^{-2 l p} .
$$


This gives

$$
\int_{\mathbf{R}^{n}}\left|\phi_{i j}(x, y)\right|^{p} d x \leq A(l, n, p) C \int_{0}^{\infty} s^{(2 l-1) p-1}(s+y)^{-2 l p} d s=A(l, n, p) C y^{-p},
$$

and the first induction step is proved.

Assume that the statement is true for $k-1$. Then $M_{p}\left(y, \nabla^{k-1} F\right) \leq A C y^{-(k-1)}$. To prove it for $k$ we define $l$ as above and apply Corollary 1 with $b=k-1, m=1$, $u=\nabla^{k-1} F$.

Lemma 3. Let $D_{i} U \Rightarrow_{y \rightarrow \infty}^{x} 0, i=1, \ldots, n$, and let

$$
M_{p}\left(y, D_{n+1} U\right) \leq C y^{-1}
$$

for some $p>0$. Then

$$
\int_{\mathbf{R}^{n}}\left(\sup _{y>0}\left|D_{n+1} U\left(x, y+y_{0}\right)\right|\right)^{p} d x \leq A C, \quad \forall y_{0}>0, \quad A=A\left(n, p, y_{0}\right) .
$$

Proof. Let $p>1$. Then (see [12])

$$
\left\|\sup _{y>0}\left|D_{n+1} U\left(\cdot, y+y_{0}\right)\right|\right\|_{p} \leq C M_{p}\left(y+y_{0}, D_{n+1} U\right) \leq A C, \quad A=A\left(n, p, y_{0}\right) .
$$

Now let $0<p \leq 1$. Assume that

$$
\int_{0}^{\infty} \int_{\mathbf{R}^{n}} s^{p-1}\left|\nabla^{2} U\left(x, s+y_{0}\right)\right|^{p} d x d s \equiv \int_{0}^{1} \int_{\mathbf{R}^{n}}+\int_{1}^{\infty} \int_{\mathbf{R}^{n}}<C\left(y_{0}\right)<\infty .
$$

Then Lemma 2, Theorem 2 (with $D_{n+1} U$ instead of $u$ and $k=1$ ), and the tensor representation of $D_{n+1} U$ from [4, imply that $D_{n+1} U\left(x, y+y_{0}\right) \in H^{p}$. This gives (6).

Thus, we have to show (7). By Theorem 3, (5) yields

$$
M_{p}\left(y, \nabla^{2} U\right) \leq A C y^{-2} .
$$

Then, the first integral in the right-hand side of (7) is finite, since

$$
M_{p}\left(y+y_{0}, \nabla^{2} U\right) \leq A C\left(y+y_{0}\right)^{-2} \leq A C\left(y_{0}\right), \quad \forall y_{0}>0 .
$$

On the other hand,

$$
\int_{1}^{\infty} s^{p-1} d s \int_{\mathbf{R}^{n}}\left|\nabla^{2} U\left(x, s+y_{0}\right)\right|^{p} d x \leq A C \int_{1}^{\infty} s^{p-1}\left(s+y_{0}\right)^{-2 p} d s \leq A C\left(y_{0}\right)<\infty .
$$

Let $V_{i}$ be components of the harmonic vector $F=\left(U, V_{1}, \ldots, V_{n}\right)$. By the meanvalue theorem,

$$
V_{i}(x, y)=V_{i}(x, y+1)-D_{n+1} V_{i}\left(x, y+\theta_{i}\right), \quad 0<\theta_{i}(x, y)<1 .
$$

Lemma 4. Let $F=\left(U, V_{1}, \ldots, V_{n}\right) \in H^{q}$ and let $\theta_{i}$ be as in (9) $, i=0, \ldots, n$. Then

$$
\int_{\mathbf{R}^{n}}\left(\sup _{y>0}\left|D_{i} U\left(x, y+\theta_{i}\right)\right|\right)^{q} d x \leq A C .
$$


Proof. We have

$$
\begin{gathered}
\sup _{y>0}\left|V_{i}(x, y)\right| \leq \sup _{y>0}\left|V_{i}(x, y+1)\right|+\sup _{y>0}\left|D_{n+1} V_{i}\left(x, y+\theta_{i}\right)\right|, \\
\sup _{y>0}\left|D_{n+1} V_{i}\left(x, y+\theta_{i}\right)\right| \leq 2 F^{+}(x),
\end{gathered}
$$

where $i=0, \ldots, n, V_{0}=U$. Then, (12) implies that

$$
\int_{\mathbf{R}^{n}}\left(\sup _{y>0}\left|D_{n+1} V_{i}\left(x, y+\theta_{i}\right)\right|\right)^{q} d x \leq A C .
$$

To get the desired result we apply the Cauchy-Riemann equations $D_{i} U(x, y)=$ $D_{n+1} V_{i}(x, y)$.

In the following result we observe that $\theta_{i}>0$ on a set, controlled by estimate (2). Then we use the fact that under conditions (14) the supremum $\sup _{y>0}|F(x, y)|$ is attained at the boundary.

Theorem 5. Let $0<p<q$ and let $F=\left(U, V_{1}, \ldots, V_{n}\right)$ be a harmonic vector in $\mathbf{R}_{+}^{n+1}$. Then $F \in h^{p}$, provided

$$
\text { 1) } M_{p}(y+1, F) \leq C
$$

2) $M_{p}\left(y, D_{n+1} U\right) \leq C y^{-1}$

3) $F \in H^{q}$.

Moreover, $F \in H^{r} \forall r, p \leq r \leq q$, if and only if conditions (14) are valid.

Proof. We prove at first that (14) implies $F \in h^{p}$. Let $\theta_{i}$ be as in (9). Due to 2), (6), and 1), it is enough to show that $\sup _{y>0}\left|D_{n+1} V_{i}\left(\cdot, y+\theta_{i}(\cdot, y)\right)\right| \in L^{p}\left(\mathbf{R}^{n}\right)$. This will follow from the Cauchy-Riemann equations and

$$
\int_{\mathbf{R}^{n}}\left(\sup _{y>0}\left|D_{i} U\left(x, y+\theta_{i}(x, y)\right)\right|\right)^{p} d x<\infty .
$$

Thus, we prove (15). Condition 3) and Lemma 1 imply that

$$
\sup _{x \in \mathbf{R}^{n}}\left|D_{i} U\left(x, y+\theta_{i}\right)\right| \leq \sup _{x \in \mathbf{R}^{n}} \frac{A C}{\left(y+\theta_{i}\right)^{1+n / q}} \leq A C\left(y+\alpha_{i}(y)\right)^{-1-n / q},
$$

where $\alpha_{i}(y)=\inf _{x \in \mathbf{R}^{n}} \theta_{i}(x, y)$. Define

$$
L_{i}=\left\{x \in \mathbf{R}^{n}: \sup _{y>0}\left|D_{i} U\left(x, y+\theta_{i}\right)\right| \leq \sup _{y>0} \frac{A C}{\left(y+\alpha_{i}(y)\right)^{1+n / q}} \leq 1\right\} .
$$

Then $\forall x \in C L_{i}$ (the complement of $L_{i}$ ) we have $\sup _{y>0}\left|D_{i} U\left(x, y+\theta_{i}\right)\right|>1$. Then

$$
\int_{C L_{i}}\left(\sup _{y>0}\left|D_{i} U\left(x, y+\theta_{i}(x, y)\right)\right|\right)^{p} d x \leq \int_{\mathbf{R}^{n}}\left(\sup _{y>0}\left|D_{i} U\left(x, y+\theta_{i}(x, y)\right)\right|\right)^{q} d x \leq A C
$$

due to condition 3) and Lemma 4,

We estimate the integral in (15) over $L_{i}$. Observe that for fixed $x \in L_{i}$,

$$
\sup _{y>0}\left|D_{i} U\left(x, y+\theta_{i}(x, y)\right)\right| \leq \sup _{y>0}\left|D_{i} U\left(x, y+\alpha_{i}(y)\right)\right|,
$$


and $\alpha_{i} \equiv \inf _{y>0} \alpha_{i}(y)>0$. We put $\gamma=\min _{i=0, \ldots, n} \alpha_{i}>0$, and take any $0<y_{0} \leq \gamma$. Then

$$
\int_{L_{i}}\left(\sup _{y>0}\left|D_{i} U\left(x, y+\theta_{i}(x, y)\right)\right|\right)^{p} d x \leq \int_{\mathbf{R}^{n}}\left(\sup _{y>0}\left|\nabla U\left(x, y+y_{0}\right)\right|\right)^{p} d x \leq A C .
$$

Indeed, condition 3) and Theorem 3imply that $V_{i}(x, y) \Rightarrow_{y \rightarrow \infty}^{x} 0$. The same is true for all partial derivatives of $V_{i}, i=0, \ldots, n, V_{0}=U$. Now the second inequality in (19) follows from 2) and Lemma 3 . Taking into account (19), (18), we get (15). Thus, $F \in h^{p}$.

Now, $F \in H^{r} \forall r: p \leq r \leq q$, implies (14) by Theorem 3. We prove the converse statement. It is enough to show that $F \in H^{p}$, or $\left(\sup _{y>0}|F(\cdot, y)|\right)^{p} \in L^{1}\left(\mathbf{R}^{n}\right)$. Since $F \in h^{p}$, we apply the Fatou Lemma to obtain

$$
\int_{\mathbf{R}^{n}}|F(x, 0)|^{p} d x=\int_{\mathbf{R}^{n}}\left(\lim _{y \rightarrow 0}|F(x, y)|\right)^{p} d x \leq \lim _{y \rightarrow 0} M_{p}(y, F) \leq C .
$$

We claim that $\sup _{y>0}|F(x, y)|=|F(x, 0)|$ and our result follows from (20). Since for fixed $x_{0} \in \mathbf{R}^{n}$ the function $W F\left(x_{0}, y\right) \equiv \sup _{\eta \geq y}\left|F\left(x_{0}, \eta\right)\right|$ is nonincreasing in $y$, we have

$$
\sup _{y>0}\left|F\left(x_{0}, y\right)\right|=\sup _{y>0} \sup _{\eta \geq y}\left|F\left(x_{0}, \eta\right)\right|=\lim _{y \rightarrow 0} \sup _{\eta \geq y}\left|F\left(x_{0}, \eta\right)\right|=\left|F\left(x_{0}, 0\right)\right| .
$$

Even if $\sup _{y>0}\left|F\left(x_{0}, y\right)\right|=\left|F\left(x_{0}, y_{0}\right)\right|$ for some $y_{0}>0$, then $W F\left(x_{0}, y\right)=W F\left(x_{0}, y_{0}\right)$ for all $0 \leq y \leq y_{0}$, and we may put $\left|F\left(x_{0}, y_{0}\right)\right|=\left|F\left(x_{0}, 0\right)\right|$.

Theorem 6. Let $0<p<q, k \in \mathbf{N}$, and let $F=\left(U, V_{1}, \ldots, V_{n}\right)$ be a harmonic vector in $\mathbf{R}_{+}^{n+1}$. Then $F \in H^{r} \forall r, p \leq r \leq q$, if and only if

$$
\text { 1) } M_{p}(y+1, F) \leq C
$$

2) $M_{p}\left(y, D_{n+1}^{k} U\right) \leq C y^{-k}$

3) $F \in H^{q}$.

Proof. By Corollary 1] and the inverse statement, proved in 9], conditions (14), (21) are equivalent.

Theorem 7. Let $p>0$ and let $F=\left(U, V_{1}, \ldots, V_{n}\right)$ be a harmonic vector in $\mathbf{R}_{+}^{n+1}$ such that

$$
\text { 1) } M_{p}(y+1, F) \leq C, \quad \text { 2) } M_{p}(y, U) \leq C, \quad \text { 3) }|U(x, y)| \leq C .
$$

Then $F \in H^{r}, r \geq p$.

Proof. By Theorem 4 we have $F \in H^{r}, r>p$. Let $r=p$. By Lemma 1 and Theorem 3 we have $M_{p}\left(y, \nabla^{k} U\right) \leq C y^{-k}$, and we may use Theorem 6 .

Lemma 5. Let $0<p<q$ and let $F=\left(U, V_{1}, \ldots, V_{n}\right)$ be a harmonic vector in $\mathbf{R}_{+}^{n+1}$ such that

$$
\text { 1) } M_{p}(y+1, F) \leq C, \quad \text { 2) } \int_{\mathbf{R}^{n}}\left(\sup _{\eta \geq y}\left|D_{n+1} U(x, \eta)\right|\right)^{q} d x \leq C .
$$

Then $F \in H^{q}$. 
Proof. By the mean-value theorem,

$$
\sup _{y>0}|U(x, y)| \leq \sup _{y>0}|U(x, y+1)|+\sup _{y>0}|\nabla U(x, y)|,
$$

and it is enough to show that $\left(\sup _{y>0}|U(\cdot, y+1)|\right)^{p} \in L^{1}$. To prove this, we apply Theorem 3 the mean-value theorem again,

$$
|U(x, y+1)| \leq|U(x, y+2)|+\sup _{y>0}\left|D_{n+1} U(x, y+1+\theta)\right|,
$$

and observe that the conditions of Theorem 7 are satisfied with $y+1$ instead of $y$.

\section{Proof of Theorem 1}

The proof is given in two lemmata presented below.

Lemma 6. Let $k \in \mathbf{N}, 0<p<q$ and let $F=\left(U, V_{1}, \ldots, V_{n}\right)$ be a harmonic vector in $\mathbf{R}_{+}^{n+1}$. Then $F$ and all its partial derivatives up to order $k$ belong to $H^{r}$, $p \leq r \leq q$, if and only if

$$
\text { 1) } \left.M_{p}\left(y+y_{0}, F\right) \leq C\left(y_{0}\right) \forall y_{0}>0, \quad 2\right) \int_{\mathbf{R}^{n}}\left(\sup _{\eta \geq y}\left|D_{n+1}^{k} U(x, \eta)\right|\right)^{q} d x \leq C .
$$

Proof. The only if part is trivial. We prove if by induction. Let $k=1$. We show at first that $F \in H^{r}, p \leq r \leq q$. By Lemma 5 we have $F \in H^{q}$, and it is enough to show that $F \in H^{p}$. To this end, we apply the mean-value theorem and repeat the proof of Theorem 5 beginning with (15). As in Theorem 5 we define $L_{i}$ (see (17)), and (18) follows from 2). The last estimate in (19) follows from Theorem 3 and Lemma 7. Conditions (22) are satisfied with $\nabla U$ instead of $F, D_{n+1} U$ instead of $U$, and $y+y_{0}$ instead of $y$.

To show that all partial derivatives of the first order belong to $H^{r}, p \leq r \leq q$, one has to proceed as above by changing $\nabla U$ by $\nabla^{2} U$, and $D_{n+1}^{2} U$ by $D_{n+1} U$.

Assume that the statement is true for $k-1$, and we have to prove it for $k$. By Theorem 3 we have 1) with $D_{n+1}^{k-1} U$ instead of $F$, and the result follows.

Lemma 7. The conditions of the theorem are equivalent to the conditions of the previous lemma.

Proof. It is enough to prove that $M_{p}(y+1, F) \leq C$ and 2) of (24) imply 1) of (24). This will follow from $F \in H^{q}$. Since $V_{i}(x, y) \Rightarrow_{y \rightarrow \infty}^{x} 0, i=1, \ldots, n$, it is enough to show that $U \in H^{q}$. We will subsequently show that all $D_{n+1}^{k-1} U, D_{n+1}^{k-2} U, \ldots, U \in H^{q}$. In fact, we prove that $D_{n+1}^{k-1} U \in H^{q}$. The proof of $D_{n+1}^{k-2} U, \ldots, U \in H^{q}$ is similar.

Observe that $D_{n+1}^{k-1} U \in H^{q}$ follows from $D_{n+1}^{k-1} U \in h^{q}$. Indeed, let $D_{n+1}^{k-1} U \in h^{q}$. By the mean-value theorem we have

$$
\sup _{y>0}\left|D_{n+1}^{k-1} U(x, y)\right| \leq \sup _{y>0}\left|D_{n+1}^{k-1} U(x, y+1)\right|+\sup _{y>0}\left|D_{n+1}^{k} U(x, y)\right|,
$$

and we may apply Lemmal7 ( $w i t h p=q, D_{n+1}^{k-1} U(x, y+1)$ instead of $U(x, y), \nabla^{k-1} F$ instead of $F$ ) to obtain

$$
\int_{\mathbf{R}^{n}}\left(\sup _{y>0}\left|D_{n+1}^{k-1} U(x, y+1)\right|\right)^{p} d x<\infty .
$$


The assumption $D_{n+1}^{k-1} U \in h^{q}$ and Lemma 1 are used to satisfy the third condition of Lemma 7. The above inequality gives $D_{n+1}^{k-1} U \in H^{q}$.

Thus, it remains to prove that $D_{n+1}^{k-1} U(x, y) \in h^{q}$. By the mean-value theorem,

$$
\left|D_{n+1}^{k-1} U(x, y)\right| \leq\left|D_{n+1}^{k-1} U(x, y+1)\right|+\sup _{y>0}\left|D_{n+1}^{k} U(x, y)\right|
$$

and it is enough to prove that $D_{n+1}^{k-1} U(x, y+1) \in h^{q}$. Again, by the mean-value theorem,

$$
\left|D_{n+1}^{k-1} U(x, y+1)\right| \leq\left|D_{n+1}^{k-1} U(x, y+1+1)\right|+\sup _{y>0}\left|D_{n+1}^{k} U(x, y)\right|,
$$

but now we may use the assumption $M_{p}(y+1, F) \leq C$ to show that for $r \geq p$, $M_{r}\left(y+2, D_{n+1}^{k-1} U\right) \leq C$. To this end, we apply Theorem 13, Lemma 1, and take $y+1$ instead of $y$.

\section{REFERENCES}

[1] A. A. Bonami, Integral inequalities for conjugate harmonic functions of several variables, Math. Sbornik, Vol. 87129 (1972), No. 2, pp. 188-203. MR0299818(45:8866)

[2] D. L. Burkholder, R. V. Gundy and M. L. Silverstein, A maximal function characterization of the class $H^{p}$, Trans. AMS., 157 (1971), 137-153. MR0274767(43:527)

[3] A. P. Calderón and A. Zygmund, On higher gradients of harmonic functions, Studia Math. 24 (1964), No. 2, 211-266. MR0167631 (29:4903)

[4] C. Fefferman and E. M. Stein, $H^{p}$ spaces of several variables, Acta Math., 129, No. 3, (1972), 137-193. MR0447953(56:6263)

[5] T. M. Flett, Inequalities for the p-th mean values of harmonic and subharmonic functions with $p \leq 1$, Proc. London Math. Soc., Ser. 3, 20, No. 3, (1970), 249-275. MR0257387 $(41: 2038)$

[6] V. I. Krylov, On functions regular in the half-plane, Math., Sb., (1939), 6 (48), pp.95-138.

[7] U. Kuran, Classes of subharmonic functions in $\mathbf{R}^{n} \times(0, \infty)$, Proc. London Math. Soc., Ser. 3, 16 (1966), No. 3, 473-492. MR0203059 (34:2917)

[8] I. Privalov, Subharmonic functions, Moscow, 1937.

[9] A. Ryabogin, Conjugate harmonic functions of the Hardy class. (Russian), Izv. Vyssh. Uchebn. Zaved. Mat. 1991, , no. 9, 47-53; translation in Soviet Math. (Iz. VUZ) 35 (1991), no. 9, 46-51. MR 1169391 (93e:42033)

[10] A. Ryabogin, Boundary values of conjugate harmonic functions of several variables, (Russian), Izv. Vyssh. Uchebn. Zaved. Mat. (1980), no. 12, 50-54. MR606677 (82d:31007)

[11] E. D. Solomentsev, On classes of subharmonic functions in the half-space, Notes of Moscow State Univ., 10, (1958).

[12] E. M. Stein, Singular integrals and differentiability properties of functions, Princeton University Press, Princeton NJ, 1970. MR0290095 (44:7280)

[13] E. M. Stein and G. Weiss, On the theory of harmonic functions of several variables, Acta Math., 103 (1960), pp. 26-62. MR0121579 (22:12315)

[14] E. M. Stein and G. Weiss, Generalization of the Cauchy-Riemann equations and representation of the rotation group, Amer. J. Math., 90 (1968), 163-196. MR0223492 (36:6540)

[15] E. M. Stein and G. Weiss, An introduction to harmonic analysis on Euclidean spaces, Princeton University Press, Princeton NJ, 1969.

[16] T. Wolff, Counterexamples with harmonic gradient in $\mathbf{R}^{3}$, Essays in honor of E. M. Stein, Princeton Mathematical Series, 42 (1995), 321-384. MR 1315554 (95m:31010)

[17] A. Zygmund, Trigonometric series, 2nd ed., Cambridge University Press, Cambridge, 1968. MR0236587 (38:4882) 
Department of Mathematics, Ben Gurion University of the Negev, P.O.B. 653, Be'er SHEVA 84105, IsRAEL

E-mail address: ryabs@math.ksu.edu

Department of Mathematics, Kansas State University, Manhattan, Kansas 665062602

E-mail address: ryabs@math.ksu.edu 